\title{
Thermal Characteristics of Foils for an Imaging Bolometer*)
}

\author{
Ryuichi SANO, Byron J. PETERSON ${ }^{1)}$, Evgeny A. DRAPIKO ${ }^{1)}$, Yuto WATANABE ${ }^{2)}$, \\ Yuji YAMAUCHI and Tomoaki HINO \\ Hokkaido University, Kita-8, Nishi-5, Kita-ku, Sapporo 060-0808, Japan \\ 1) National Institute for Fusion Science, 322-6 Oroshi-cho, Toki 509-5292, Japan \\ ${ }^{2)}$ Tsuyama National College of Technology, 624-1 Numa, Tsuyama 708-8509, Japan
}

(Received 7 December 2010 / Accepted 21 April 2011)

\begin{abstract}
The IR imaging video bolometer is an imaging bolometer which provides the intensity and distribution of plasma radiation. The sensitivity of the IR imaging bolometer is dependent on the properties of the bolometer foil. An evaluation of the thermal characteristics of various materials and thicknesses of the bolometer foil provides information on the sensitivity which is useful to choose the best foil material. We irradiated foils of various materials and thicknesses with a He-Ne laser (wavelength $633 \mathrm{~nm}$ ), and measured the change in temperature distribution with an IR camera. As for foil materials, W, Ta, Au and Pt were employed. The foils were blackened either on both sides or on one side by graphite. For the same material foil, the temperature rise in the singleside blackened foil was always greater than the double-side blackened foil. For the double blacken foil, Ta had the largest temperature rise among foils with the same thickness. Pt had the shortest time constant for the temperature rise/decayamong foils except $\mathrm{Au}$. In consideration of the attenuation thickness versus photon energy of each material, the Pt foil was the most suitable for the bolometer among the evaluated materials.
\end{abstract}

(c) 2011 The Japan Society of Plasma Science and Nuclear Fusion Research

Keywords: imaging bolometer, plasma diagnostics

DOI: $10.1585 /$ pfr.6.2406076

\section{Introduction}

An IR imaging video bolometer (IRVB) [1] is a measuring instrument for plasma radiation. It is useful for the measurement of radiation intensity and distribution from plasma. These observations are useful for operation of a fusion device. IRVBs have been used in LHD [2] and JT60 [3] with thin bolometer foils $(2.5 \mu \mathrm{m})$. However, improved ability by the IRVBto stop high energy photonsby is required for future use on next-generation fusion devices such as ITER. For this improvement it is necessaryto increase the bolometer foil thickness. In addition, the performance of an IRVB is dependent on the thermal characteristics and photon absorptive property of the bolometer foil. The bolometer foil was blackened by graphite spray to improve the observation with an IR camera. Therefore, evaluation of the thermal characteristics of the blackened bolometer foils made from candidate materials is important. The foil evaluation technique was developed with a He-Ne laser [4] or ultraviolet diodes [5]. A comparison of thin Au andPt foils has been made [6]. In that study, the Pt foil has higher sensitivity than the Au foil. However, foils of other thicknesses and materials have not been evaluated systematically. The purpose of this study is the relative comparison between foils of various materials and thicknesses.

author's e-mail: doreiko@frontier.hokudai.ac.jp

*) This article is based on the presentation at the 20th International Toki Conference (ITC20).
When the bolometer foil absorbs radiation power, a 2D temperature distribution is formed on the foil. This distribution is measured by an IR camera to observe the plasma radiation. The plasma radiation power is given in terms of the temperature distribution on the foil by the following heat diffusion equation, [Eq. (1)]

$$
\begin{aligned}
-\Omega_{\mathrm{rad}}+\Omega_{\mathrm{bb}}+\frac{1}{\kappa} \frac{\partial T}{\partial t}=\frac{\partial^{2} T}{\partial x^{2}}+\frac{\partial^{2} T}{\partial y^{2}}, \\
\Omega_{\mathrm{rad}}=\frac{P_{\mathrm{rad}}}{k t_{\mathrm{f}}}, \quad \Omega_{\mathrm{bb}}=\frac{\varepsilon \sigma_{\mathrm{SB}}\left(T^{4}-T_{0}^{4}\right)}{k t_{\mathrm{f}}} .
\end{aligned}
$$

Where $T$ is the two-dimensional temperature distribution on the foil measured by the IR camera, $k$ is the foil thermal conductivity, $t_{\mathrm{f}}$ is the foil thickness, $\sigma_{\mathrm{SB}}$ is the StefanBoltzmann constant, $\varepsilon$ is the black body emissivity, $T_{0}$ is the back ground temperature and $\kappa$ is the thermal diffusivity of the foil.

The inverse of the sensitivity of the imaging bolometer is given by the noise equivalent power density (NEPD), $S_{\text {IRVB }}$. The NEPD is given by the following equation, [Eq. (2)] [7]

$$
S_{\mathrm{IRVB}}=\frac{\sqrt{2} k t_{\mathrm{f}} \sigma_{\mathrm{IR}}}{\sqrt{f_{\mathrm{IR}} N_{\mathrm{IR}}}} \sqrt{\frac{5 N_{\mathrm{bol}}^{3} f_{\mathrm{bol}}}{A_{\mathrm{f}}^{2}}+\frac{N_{\mathrm{bol}} f_{\mathrm{bol}}^{3}}{\kappa^{2}}},
$$

where $\sigma_{\mathrm{IR}}$ is the temperature resolution of the IR camera, $A_{\mathrm{f}}$ is the area of bolometer foil, $N_{\mathrm{IR}}$ is the number of pixels of the IR camera, $f_{\mathrm{IR}}$ is the frame rate of the IR camera and $f_{\text {bol }}$ is the frequency response of the imaging bolometer. 
The determining parameters for the sensitivity of the IRVB are the properties of the foil material, the performance of the IR camera and the pixel size of imaging bolometer. In terms of the foil materials the sensitivity depends on $k, \kappa$, $t_{\mathrm{f}}$. Also the foil temperature rise, $\Delta T$, and the time constant for the temperature rise/decay, $\tau$, with incidence radiation are estimated by [Eq. (3)] for the case of weak radiation cooling.

$$
\frac{1}{S_{\text {IRVB }}} \propto \frac{\kappa}{k t_{\mathrm{f}}} \propto \frac{\Delta T}{\tau} .
$$

Therefore, evaluation of $\Delta T / \tau$ provides information which is useful to choose the best foil material.

\section{Experiment}

In this experiment, we heated the foil with a chopped He-Ne laser through a Pyrex window and measured the temperature distribution change at the foil surface by an Omega IR camera (microbolometer type, manufactured by INDIGO, wavelength 7.5-13.5 $\mu \mathrm{m}, 30 \mathrm{fps}$, $120 \times 160$ pixels) with a ZnSe IR window at room temperature. The power of the He-Ne laser (wavelength $633 \mathrm{~nm}$ ), is about $12 \mathrm{~mW}$ at the foil. The period of laser chopping is $12 \mathrm{~s}$ (6s irradiation and $6 \mathrm{~s}$ chopped).

We employed $\mathrm{Au}, \mathrm{Pt}$, Ta and $\mathrm{W}$ as evaluated foil materials since they are the most likely candidate materials for an imaging bolometer foil because of their ability to stop high energy photons [8]. Because the Au foil has a high neutron cross-section which leads to transmutation to $\mathrm{Hg}$, it is not suitable for the bolometer foil of a fusion reactor. However, we employed it as an object of comparison. The foils are mounted in copper gaskets as shown in Fig. 1. The gasket is mounted in a vacuum chamber which is evacuated to less than 0.1 mTorr to remove the heat conduction cooling of the foil by air. The nominal thicknesses of foils are $\operatorname{Au}(1,2.5,3,5,7,10 \mu \mathrm{m}), \operatorname{Pt}(1,2.5,5,7.5$, $10 \mu \mathrm{m}), \mathrm{Ta}(5,7.5,10 \mu \mathrm{m})$ and $\mathrm{W}(10 \mu \mathrm{m})$. However, the average foil thicknesses calculated by foil weight, which were measured by microbalance, differ from the nominal thicknesses. In this experiment,the thicknesses of the foils, $t_{\mathrm{f}}$, used the average values shown in Table 1 . These foils are blackened on a single side or on both sides by graphite spray to increase the IR emissivity of the foil for observation by an IR camera and to increase the absorption for heating by a He-Ne laser. The emissivity of the foil material and graphite is not measured. The point which was irradiated by the He-Ne laser is the foil center of the blackened side. The IR camera observation side is the blackened side. We calibrated the IR camera output using a carbon plate which is attached to a thermo couple and a heat source and also by usinga black body calibration source.

Figure 2 shows the temperature change of the foil at the irradiation point under the incidence of a chopped He$\mathrm{Ne}$ laser. We calculated the temperature rise, $\Delta T$, and the time constant, $\tau$, for temperature rise/decay as the thermal characteristics. These parameter are calculated by curve

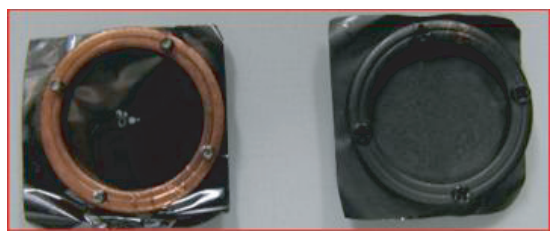

Fig. 1 The foil mounted in copper gasket. Left foil is single sided blackened foil. Right foil is double sided blackened foil.

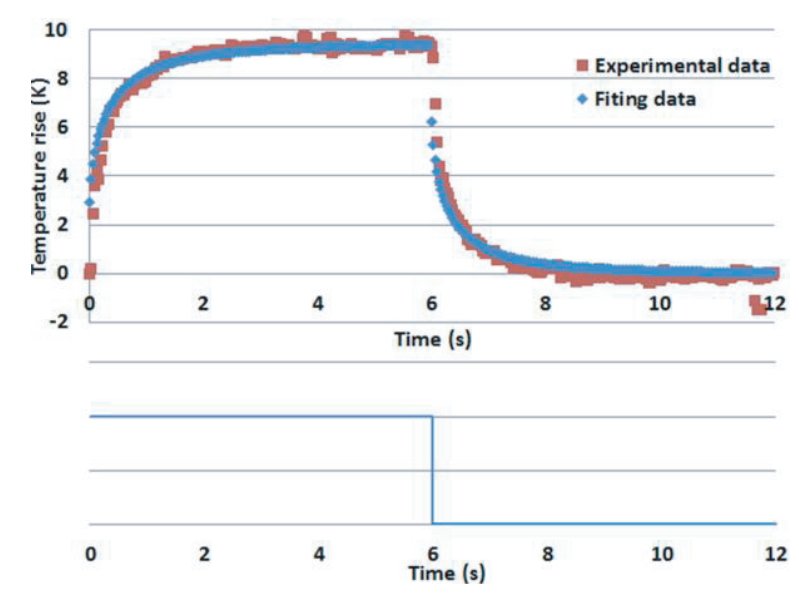

Fig. 2 Temperature rise from room temperature at the center of the foil and fit to Eq. 3 (blue) (upper) and He-Ne laser pulse (lower).

Table 1 Thickness of evaluated foils, $t_{\mathrm{f}}$ a) nominal, b) average (from weight), c) local (from SEM), d) variance of local thickness (from SEM), e) thermal conductivity, $k$ [7], and (f) thermal diffusivity, $\kappa$ [7], of foil and (g) relative material sensitivity $(k / \kappa)$. Au, Pt, Ta and $\mathrm{W}$ : double side blackened foil, $\mathrm{Au}^{*}, \mathrm{Pt}^{*}, \mathrm{Ta}^{*}$ and $\mathrm{W}^{*}$ : single side blackend foils.

\begin{tabular}{|c|c|c|c|c|c|c|c|}
\hline \multirow[t]{2}{*}{ Metal } & \multicolumn{4}{|c|}{$\mathrm{t}(\alpha \mu \mathrm{m})$} & \multirow{2}{*}{$\frac{e) k}{W /(m \cdot K)}$} & \multirow{2}{*}{$\begin{array}{c}\text { f) } \mathrm{K} \\
\times 10^{6} \mathrm{~s} / \mathrm{m}^{2} \\
\end{array}$} & \multirow{2}{*}{$\frac{g) \mathrm{k} / \mathrm{K}}{\mathrm{\times} \times 10^{-6} \mathrm{Wm} / \mathrm{K}}$} \\
\hline & a) Nom. & b)Ave. & c) Loc. & d) $\mathrm{Var}$. & & & \\
\hline $\mathrm{Au}$ & 1 & 0.83 & 12 & \pm 0.2 & \begin{tabular}{|r|}
318 \\
\end{tabular} & \begin{tabular}{|r}
127 \\
\end{tabular} & \begin{tabular}{|r|}
2.50 \\
\end{tabular} \\
\hline $\mathrm{Au}$ & 2.5 & 0.96 & 2.3 & \pm 0.5 & $"$ & $n$ & " \\
\hline $\mathrm{Au}$ & 3 & 2.7 & 2.5 & \pm 0.3 & $"$ & $"$ & $"$ \\
\hline $\mathrm{Au}$ & 5 & 4.88 & 6.5 & \pm 1.1 & " & $n$ & " \\
\hline $\mathrm{Au}$ & 7 & 7.14 & 6.5 & \pm 1.5 & " & $n$ & $n$ \\
\hline $\mathrm{Au}$ & 10 & 12.2 & 11.5 & \pm 1.1 & $"$ & $"$ & $"$ \\
\hline $\mathrm{Pt}$ & 1 & 0.91 & 0.9 & \pm 02 & 71.6 & 25.1 & 2.85 \\
\hline $\mathrm{Pt}$ & 3 & 0.72 & 1.3 & \pm 0.2 & $"$ & " & n \\
\hline $\mathrm{Pt}$ & 5 & 4.69 & 7.8 & \pm 1.8 & " & $n$ & $"$ \\
\hline $\mathrm{Pt}_{\mathrm{t}}$ & 7.5 & 7.21 & 7.3 & \pm 1.2 & & " & " \\
\hline $\mathrm{Pt}$ & 10 & 10.2 & 7.2 & \pm 2.0 & $"$ & " & 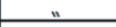 \\
\hline W & 10 & 8.32 & 7.6 & \pm 0.2 & 173 & 67.4 & 2.57 \\
\hline $\mathrm{Ta}$ & 5 & 4.46 & 4.8 & \pm 0.3 & 57.5 & 24.7 & 2.33 \\
\hline $\mathrm{Ta}_{\mathrm{a}}$ & 7.5 & 8.18 & 13.1 & \pm 2.0 & " & $n$ & n \\
\hline $\mathrm{Ta}$ & 10 & 6.79 & 9.8 & \pm 3.3 & " & " & " \\
\hline$A u^{*}$ & 10 & & 12.8 & \pm 0.8 & 318 & 127 & 2.50 \\
\hline $\mathrm{Pt}^{*}$ & 10 & & 6.8 & \pm 1.1 & 71.6 & 25.1 & 2.85 \\
\hline$W^{*}$ & 10 & & 8.4 & \pm 0.4 & 173 & 67.4 & 2.57 \\
\hline $\mathrm{Ta} a^{*}$ & 10 & & 15.2 & \pm 9.8 & 57.5 & 24.7 & 2.33 \\
\hline
\end{tabular}

fitting to [Eq. (4)].

$$
T=\Delta T(1-\exp (-\sqrt{t / \tau}))+T_{0} .
$$

The shorter the time constant and the larger the temperature rise is, the higher the sensitivityof the bolometer. 


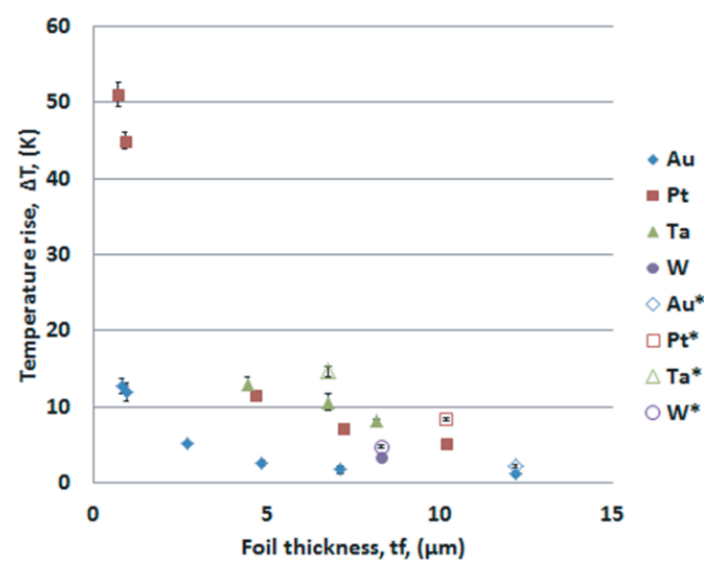

Fig. 3 The temperature rise from room temperature at center of double blacken foils (Au, Pt, Ta and W) (full symbol) and single blacken foil $\left(\mathrm{Au}^{*}, \mathrm{Pt}^{*}, \mathrm{Ta}^{*}\right.$ and $\left.\mathrm{W}^{*}\right)$ (open symbol). The error bars represent a standard deviation.

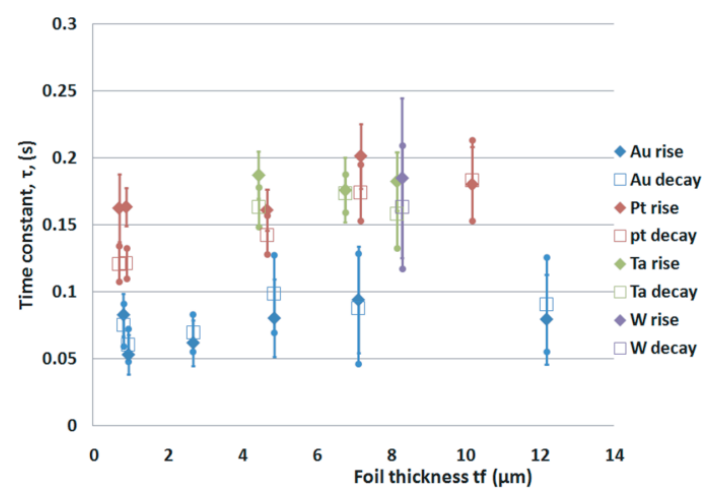

Fig. 4 The time constant for temperature rise/decay at the center of the double sided blackened foils. The error bars represent a standard deviation.

\section{Results}

\subsection{Temperature rise}

Figure 3 shows the temperature rise to steady state from room temperature at the center of the foil versusfoil thickness. For the same thickness foil, the temperature rise of the single sided blackened foils was larger than the double sided blackened foils. Also the Ta foil has the largest temperature rise among the same thickness double sided blackened foils. The temperature rise, $\Delta T$, data are scattered for the thin foil. This scattering may be due to several things. Primarily, due to the large spatial variation in the local thickness of the foil, as seen in Table 1, a slight shift of the irradiation point on each experiment may result in a small change of local thickness at irradiation point and a large change of the $\Delta T$, due to the strong dependence of $\Delta T$ on the thickness for the thin foil. Also, the laser power may change due to slight motion of the laser and spatial variations in the laser transmission of the Pyrex windows. Third, the high temperature data (large value signal) have a stronger influence on noise than low (small value signal).

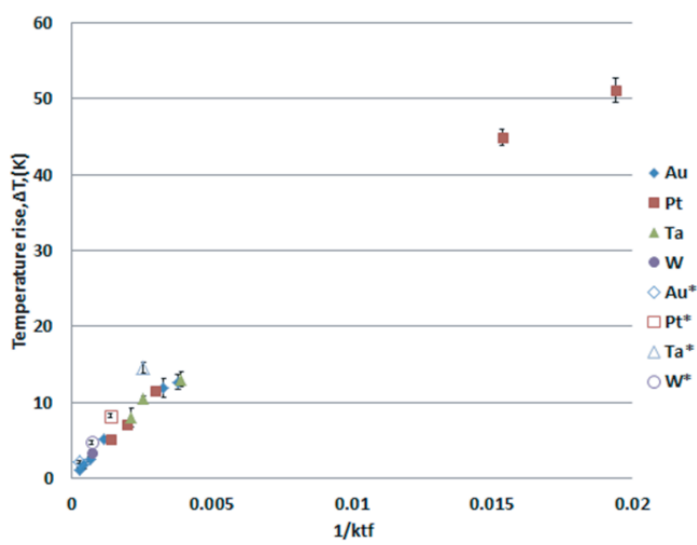

Fig. 5 The $\Delta T$ of each material versus $1 / k t_{\mathrm{f}}$. Full symbols (Au, $\mathrm{Pt}, \mathrm{Ta}$ and $\mathrm{W})$ : double blacken foils. Open symbols ( $\mathrm{Au}^{*}$, $\mathrm{Pt}^{*}, \mathrm{Ta}^{*}$ and $\left.\mathrm{W}^{*}\right)$ : single blacken foils. The error bars represent a standard deviation.

We observed the trend of $\Delta T$ by averaging over twenty experimentson each foil to exclude these influences and to get aclear relation among $\Delta T$, foil thickness and foil materials.

\subsection{Time constant for temperature rise/decay}

Figure 4 shows the time constant for the temperature rise/decay of double sided blackened foils versus foil thickness.

We observed a time constant dependence on the foil material. The time constant of each material is, in order from the shortest to the longest, $\mathrm{Au}, \mathrm{Pt}, \mathrm{Ta}$ and $\mathrm{W}$ foil. However, there is no observed strong dependence of the foil thickness upon the time constant.

\section{Discussions}

The theoretical dependence of the temperature rise, $\Delta T$, in steady state was given by solving [Eq. (1)] without the blackbody radiation term. It can be written as [Eq. (5)]

$$
\Delta T \propto \frac{1}{k t_{\mathrm{f}}},
$$

where $\Delta T$ is the temperature rise at the center of the foil from room temperature. Figure 5 shows the $\Delta T$ dependence of $1 / k t_{\mathrm{f}}$. The $\Delta T$ is in good proportion to $1 / k t_{\mathrm{f}}$ at low temperature. However, at high temperature, $\Delta T$ is smaller than the estimate from the low temperature data. It may be due to the effect of blackbody radiation cooling the foil.

Also the bolometer foil is required to absorb high energy photons from plasma radiation. Figure 6 shows the attenuation length versus photon energy and $\Delta T$ of each foil versus the upper limit of absorbable photon energy [9]. For comparison of the foils which can absorb the same energy photon, the $\Delta T$ of $\mathrm{Pt}$ is the highest among the evaluated foils. For the combined comparison of these results, we plotted Fig. 7. Figure 7 shows the absorbable photon energy of each foil versus $\Delta T / \tau_{\text {ave }}$. The $\tau_{\text {ave }}$ is the average of time constant for the temperature rise/decay. From 

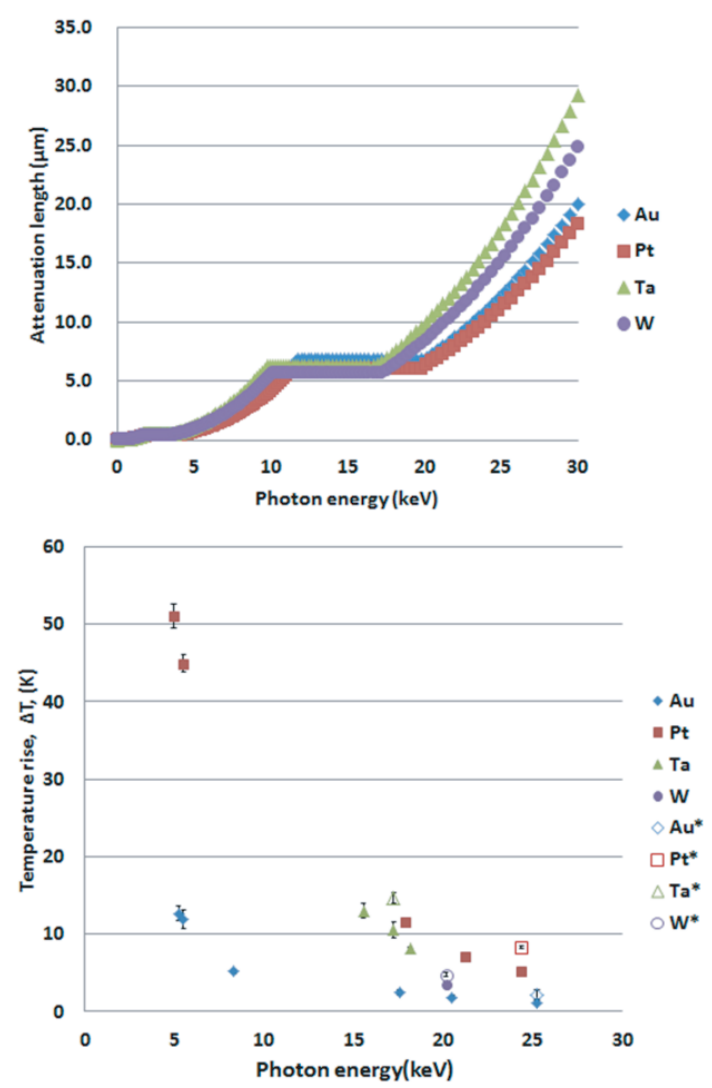

Fig. 6 The photon energy versus the necessary thickness to absorb it (upper). The $\Delta T$ of each foil versus its absorbable photon energy (lower). Full symbols ( $\mathrm{Au}, \mathrm{Pt}, \mathrm{Ta}$ and $\mathrm{W}$ ): double blacken foils. Open symbols $\left(\mathrm{Au}^{*}, \mathrm{Pt}^{*}, \mathrm{Ta}^{*}\right.$ and $\left.\mathrm{W}^{*}\right)$ : single blacken foils. The error bars represent a standard deviation.

this figure, for the foils which have same photon absorptive property, the Pt foil has the best thermal characteristics for a bolometer foil among the evaluated foil materials.

\section{Summary}

We measured the thermal characteristics of various foils. The results are as follows.

For the same thickness foils, both the Ta and Pt foils

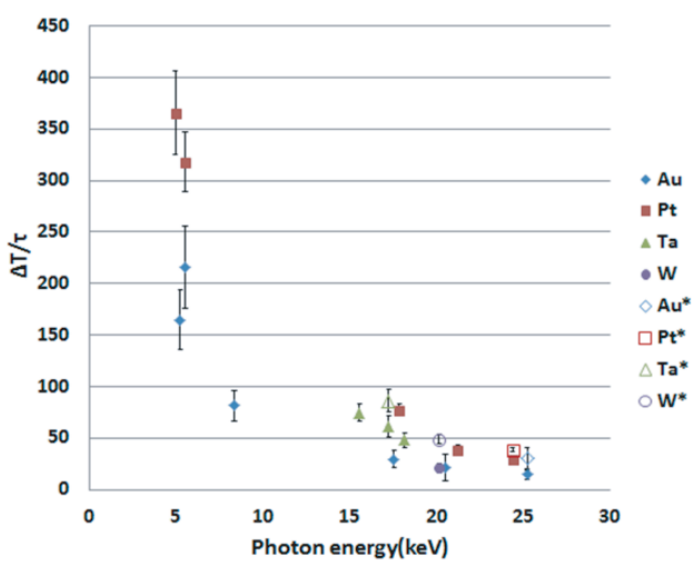

Fig. 7 The $\Delta T / \tau_{\text {ave }}$ of each foil versus its absorbable photon energy. The higher it is, the higher the sensitivity. The further to the right it is, the better the photon absorptive property. Full symbols ( $\mathrm{Au}, \mathrm{Pt}, \mathrm{Ta}$ and $\mathrm{W}$ ): double blacken foils. Open symbols $\left(\mathrm{Au}^{*}, \mathrm{Pt}^{*}, \mathrm{Ta}^{*}\right.$ and $\left.\mathrm{W}^{*}\right)$ : single blacken foils. The error bars represent a standard deviation.

have good thermal characteristics as evaluated from the foils response to a $\mathrm{He}-\mathrm{Ne}$ laser. However, for the foils which have same photon absorptive property, the Pt foil has the best thermal characteristics for an imaging bolometer foil among the evaluated foil materials.

Based on these result, the Pt foil is the most suitable for an imaging bolometer foil among the evaluated foils.

[1] B.J. Peterson et al., Rev. Sci. Instrum. 74(3), 2040 (2003).

[2] B.J. Peterson et al., Fusion Sci. Technol. 58(1), 412 (2010).

[3] B.J. Peterson et al., J. Nucl. Mater. 363-365, 412 (2007).

[4] E. Drapiko et al., Rev. Sci. Instrum. 81, 10E116 (2010).

[5] H. Parchamy et al., Rev. Sci. Instrum. 77, 10E515 (2006).

[6] B.J. Peterson et al., Plasma Fusion Res. 5, 035 (2010).

[7] B.J. Peterson et al., Rev. Sci. Instrum. 79, 10E301 (2008).

[8] B.J. Peterson et al., Plasma Fusion Res. 2, S1018 (2007).

[9] Center for X-ray Optics, Lawrence Berkeley National Laboratory, USA. http://henke.lbl.gov/optical_constants/ atten2.html 